\title{
Putting the Spotlight on Opisthorchiasis: The Dread of the Western Siberian Region
}

\author{
Aremu $\mathrm{SO}^{1,3 *}$, Zephaniah $\mathrm{HS}^{2}$, Onifade $\mathrm{EO}^{3}$, Fatoke $\mathrm{B}^{1}$ and Bademosi $\mathrm{O}^{4}$ \\ ${ }^{1}$ Faculty of General Medicine, Siberian State Medical University, Tomsk, Russian Federation \\ ${ }^{2}$ Department of Biochemistry, University of Nigeria, Nsukka, Enugu State, Nigeria \\ ${ }^{3}$ Department of Biological Science, Federal University of Agriculture, Makurdi Benue State, \\ Nigeria \\ ${ }^{4}$ Department of Public Health, University College Dublin, Ireland
}

\section{Review Article \\ Volume 4 Issue 1}

Received Date: February 17, 2020

Published Date: March 10, 2020

DOI: $10.23880 /$ phoa-16000151

*Corresponding author: Stephen Olaide Aremu, Faculty of General Medicine, Siberian State Medical University, Tomsk, Russian Federation, Email: arethomps@gmail.com

\section{Abstract}

Introduction: Opisthorchiasis is no doubt one of the most neglected infectious disease inspite of its huge medical importance in some parts of the World. The past decade have seen a resurgence of interests in research relating to this public health issue, however there is still a lot to be done.

Social Model: Not many models have been explored in Western Siberia to deal with the opisthorchiasis epidemic when compared to the different models that have been used for other regions affected by similar disease.

Life Cycle: The complex life cycle of Opisthorchis felineus has humans and other feline species as definitive host and is really prevalent among the aboriginal population of the Western Siberian because of their habit of eating raw or undercooked fresh water fish (Cyprinidae) which are intermediate host of the parasite.

Diagnosis and Treatment: Diagnosis involve the use of stool microscopy, other methods such as mAb ELISA, LAMP and so on are used, while the common treatment is the use of $25-40 \mathrm{mg}$ of the antihelminthic drug_Praziquantel.

Conclusion: The spotlight must be put on $O$. Felineus.

Keywords: Opisthorchiasis; Life Cycle; Cyprinidae; Western Siberian

\section{Introduction}

The opisthorchiidae family of the trematode class is of high economic, veterinary and medical importance, with over 45 million infected people from all round the globe [1,2]. Opisthorchis felineus, Opisthorchis viverrini, and Clonorchis sinensis, three of which belong to the aforementioned family though occasionally overlapping in terms of geographical distribution have a distinct pattern: $O$. felineus is endemic in Europe and Russia particularly the arctic region of Western Siberia; C. sinensis in China, the Republic of Korea, and northern Vietnam; and O. viverrini in Southeast Asia [1,3].
A general feature of all Opisthorchiidae is their high specificity to the first intermediate host. Typically, only one or several closely related snail species act as the first intermediate host for Opisthorchiidae, whereas the lists of second intermediate and definitive hosts may comprise up to several tens of species [4].

A study by Fedorova et al showed that in the whole of Russian Federation in 2011 to 2013, the average annual incidence of $O$. felineus infection was between the range of 15.7 to 33.7 cases per 100,000 population [5]. However, the major habitat of $O$. felineus in Russia is the Ob-Irtysh river 
basin, which spans the south to north in the Western Siberia region. Compare to the European part of Russia and Eastern Siberia, the prevalence of $O$. felineus infection in Western Siberian people has been estimated to be up to $80 \%$, about 100 to 1000 times more [5] In these regions, the prevalence rates of opisthorchiasis exceed 100 per 100000 people [6]: Khanty-Mansiiskiy Autonomous Region (600 per 100000 people), Tyumen Region (201 per 100000 people), Tomsk Region(189 per 100000 people), and Novosibirsk Region (127 per 100000 people) [6].

\section{Social Dynamics Model of Western Siberian Aboriginal population on Opisthorchiasis}

The social dynamics model of 0 . felineus transmission in Western Siberia was designed for preparing and developing an integrated opisthorchiasis control programme in the region [5]. The model attests to the specificity of transmission dynamics in Western Siberia instead of using the one size fits all approach. The model also underpins the importance of understanding this public health issue as embedded in social networks and animated by a variety of locally-specific linkages between ways of life, food and health cultures, health-care systems, economies, and landscapes $[5,6]$.

Information in different suburbs and villages of Western Siberia obtained via semi-structured interviews and questionnaires with people of indigenous background in different studies revealed that Opisthorchiasisis is a well known disease by the locals and is generally regarded as a chronic mild disease by indigenous peoples [1]. This is due to a widespread opinion in complimentary medicine that indigenous peoples have more genetic resistance to opisthorchiasis than immigrant population. An uspecified percentage of the population regard treatments (both conservative and complimentary) as more harmful than beneficial, whereas others regard them as "necessary" [2].

Knowledge of the disease does not in anyway affect the attitude of the locals to eating fish from the local river (River $\mathrm{Ob}$ ) and very frequently (as traditional staple food) and often in raw or undercooked form hence, the prevalence of opisthorchiasis is extremely high among the indigenous people $[1,6]$.

Indigenous knowledge on prevention and treatment options for opisthorchiasis include Thermal processing of fish (boiling, freezing, frying), salting. Thorough washing of hands, dishes and surfaces that have come in contact with fish and disinfection. Bogsphagnum was said to be good natural antiseptic for cleaning surfaces. Local medicine such as swallowing of gallbladder of pike, drinking extract of pinenuts in alcohol, or extract of aspen bark can help alleviate opistarchiasis.

These model summaries are: (1) that opisthorchiasis is a disease of degree, i.e. opisthorchiasis becomes a disease only when there are too many parasites in the body (infection is not equal to Disease), and (2) that use of anthelmintic drugs is not necessary in Western Siberia which is a total deviation from what is observed with chronic infection of 0 . viverreni and C. Sinensis in Northeast Asia [6] (Figure 1).

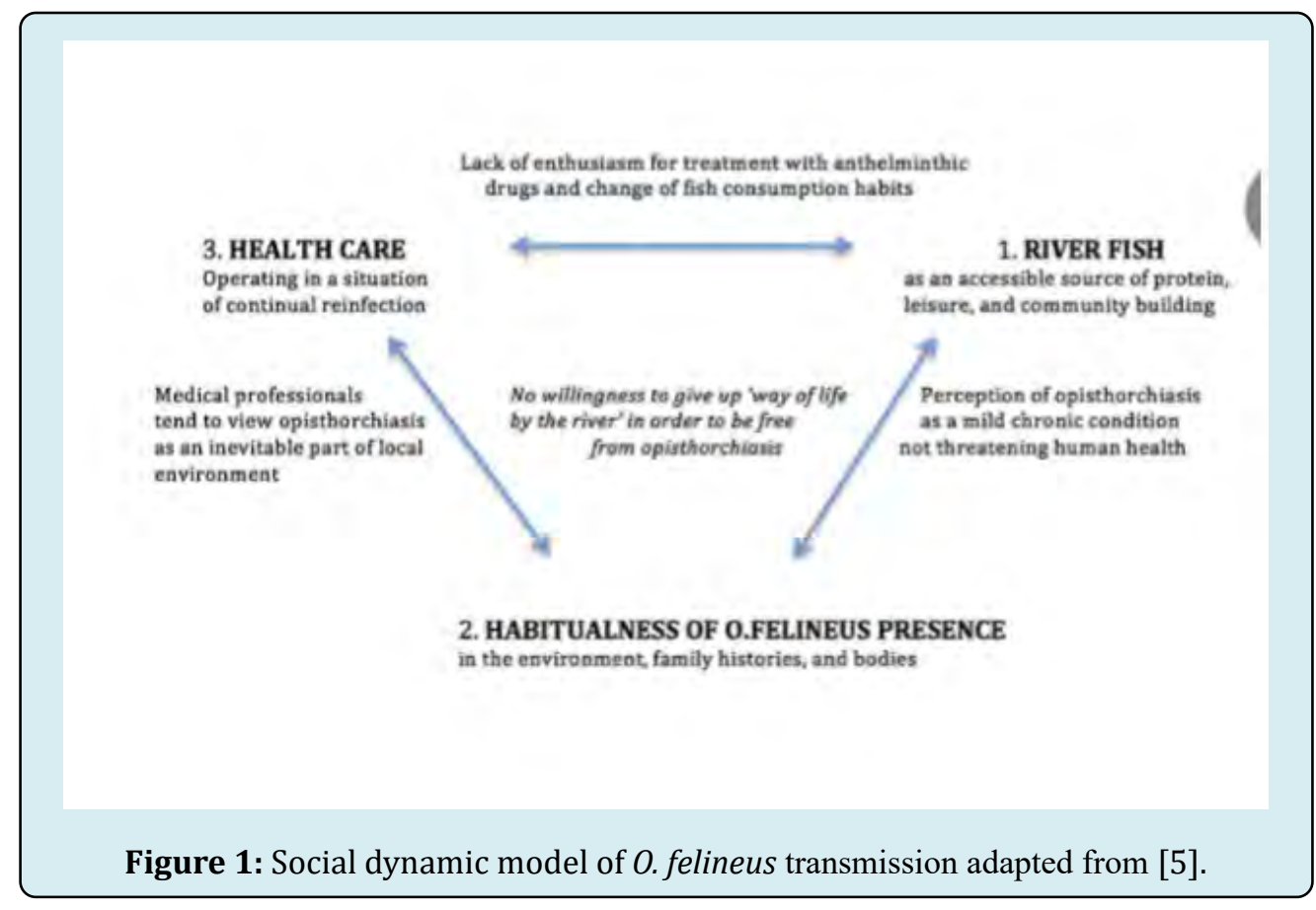

Aremu SO, et al. Putting the Spotlight on Opisthorchiasis: The Dread of the Western Siberian Region. Public H Open Acc 2020, 4(1): 000151. 


\section{Life Cycle}

Feeding from raw or undercooked fresh water fish carrying the metacercariae of $O$. felineus results in human infection $[4,7,8]$ (Table 1$)$.

\begin{tabular}{|c|c|}
\hline \multicolumn{2}{|c|}{ Opisthorchis viverrini } \\
\hline Latin name & Local name \\
\hline Cyclocheilichthys apogon & Sai Tan Ta Daeng \\
\hline Labiobarbus leptocheilus & Sa \\
\hline Puntius brevis & Taphian Jut \\
\hline Barbonymus gonionotus & Taphian Khao \\
\hline Henicorhynchus siamensis & Soi Khao \\
\hline Henicorhynchus ornatipinnis & Soi Peek Daeng \\
\hline Hampala dispar & Kra Sup Jut \\
\hline Hampala macrolepidota & Kra Sup Khit \\
\hline Osteochilus hasselti & Soi Nokkhao \\
\hline \multicolumn{2}{|c|}{ Opisthorchis felineus } \\
\hline Latin name & Common name \\
\hline Leuciscus idus & Ide \\
\hline L. cephalus & Chub \\
\hline L. leuciscus & Dace \\
\hline Rutilus rutilus & Roach \\
\hline Blicca bjorkna & Silver bream \\
\hline Carassius carassius & Crucian carp \\
\hline C. auratus gibello & Silver crucian carp \\
\hline Abramis brama & Common bream \\
\hline A. ballerus & Blue bream \\
\hline A. sapa & White-eye bream \\
\hline Chondrostoma nasus & Nase \\
\hline Scardinius erythrophtalamus & Common rudd \\
\hline Pelecus cultratus & Ziege \\
\hline Cyprinus carpio & Common or European carp \\
\hline Tinca tinca & Tench \\
\hline Gobio gobio & Gudgeon \\
\hline Alburnus alburnus & Bleak \\
\hline Phoxinus phoxinus & Minnow \\
\hline Ph. chekanowskii & Chekanovsky minnow \\
\hline Aspius aspius & Asp \\
\hline Barbus borysthenicus & Barbel \\
\hline Leucaspius delineatus & Sunbleak \\
\hline Cobitis taenia & Spined loach \\
\hline
\end{tabular}

Table 1: Local Name and Common Name of fish intermediate hosts of the Liver fluke Culled from [9].

The total list of definitive host species for this parasite comprises 33 species and subspecies of mammals, first and foremost, belonging to the order Carnivora: domestic cats, dogs, wolves, foxes, bears and badgers. Hence, it is also known as Cat Liver Flukes $[1,10]$. The ingested larvae develop further and moves to the bile ducts by chemotaxis, where the biliary epithelia and biliary contents of the gall bladder is fed upon by the adult worms. The larvae are well prepared to survive the harsh weather condition as they contain, miracidia, which allows for maintaining viability in freshwater aquatic bodies for up to 3 months if they get into these bodies in the early part of spring. Moreover, they are able to survive the period of water freezing-thawing during the overall winter and retain their viability to the July of the next summer if they entered these water bodies in the late fall [1].

After about 25-30 days, the adult worms reach sexual maturity (maritae) and there is production of numerous eggs by the adult worms which enter the gastrointestinal tract and are passed out alongside faeces to the outside environment $[1,11,2,4]$. These eggs released find their way into the waterbodies and are ingested by freshwater snails of the family Bithyniidae. Before the mid 20th century, Bithynia leachi was regarded as the only first intermediate host for 0 . felineus; later, it was discovered that there are four species: B.leachi, B.troscheli, B. inflata and B. sibirica.The first three species are susceptible to $O$. felineus.

The $O$. felineus miracidia passes through several stages of development via asexual development (transforming through sporocysts to rediae) in the snail, which is typical of all Opisthorchiidae. Plethora of free-swimming cercariae are produced from the rediae of $O$. felineus, which leave the snail for their second inter-mediate host and, following the release of the miracidia from the eggs, for the next 3 weeks to 2 months, several development stages take place within the snail until cercariae have developed. Shed cercariae can penetrate freshwater fish, encystation takes place and are localized to the subcutaneous fat and muscle tissues at a depth of 1-2 mm. However, localization to unusual sites such as gills, gut walls or pectoral fins are not uncommon [11].

The 0 . felineus metacercariae are tolerant to drying, high salt concentration, and low temperatures and can be killed only by a high temperature [1]. According to Pakharukova and Mordvinov the typical sources of $O$. felineus infection in Russia are undercooked, sun-dried, light-salted, and lightpickled fish [1]. In addition, indigenous people of Western Siberia traditionally eat cold-served slices of frozen fish [1,2].

Within the genus Opisthorchis, O. felineus species has the highest zoonotic potential, which has important implications in terms of maintenance of transmission to humans even under high hygienic standards in which the 
risk of freshwater contamination by human faeces is low as well as in vereinary medicine [12]. The morbidities associated with opisthorchiasis are largely hepatobiliary, specifically stemming from cholangiofibrosis and cholangitis, hepatobiliary inflammation, cholangiocyte dysplasia, intraepithelial neoplasia, and are expressed in a variety of manifestations, such as abdominal pain, nausea, obstructive jaundice hepatomegaly and even cholangiocarcinoma among chronically infected individuals in a pathway similar to that caused by 0 . viverrini and C. Sinensis $[2,4]$. Cholangiocarcinoma, a generally incurable and, hence, fatal bile duct cancer $[3,12]$, which has resulted in the classification of these parasites to the Group 1 carcinogens by the International Agency for Research on Cancer (IARC) especially in endemic regions [13-15] (Figure 2).

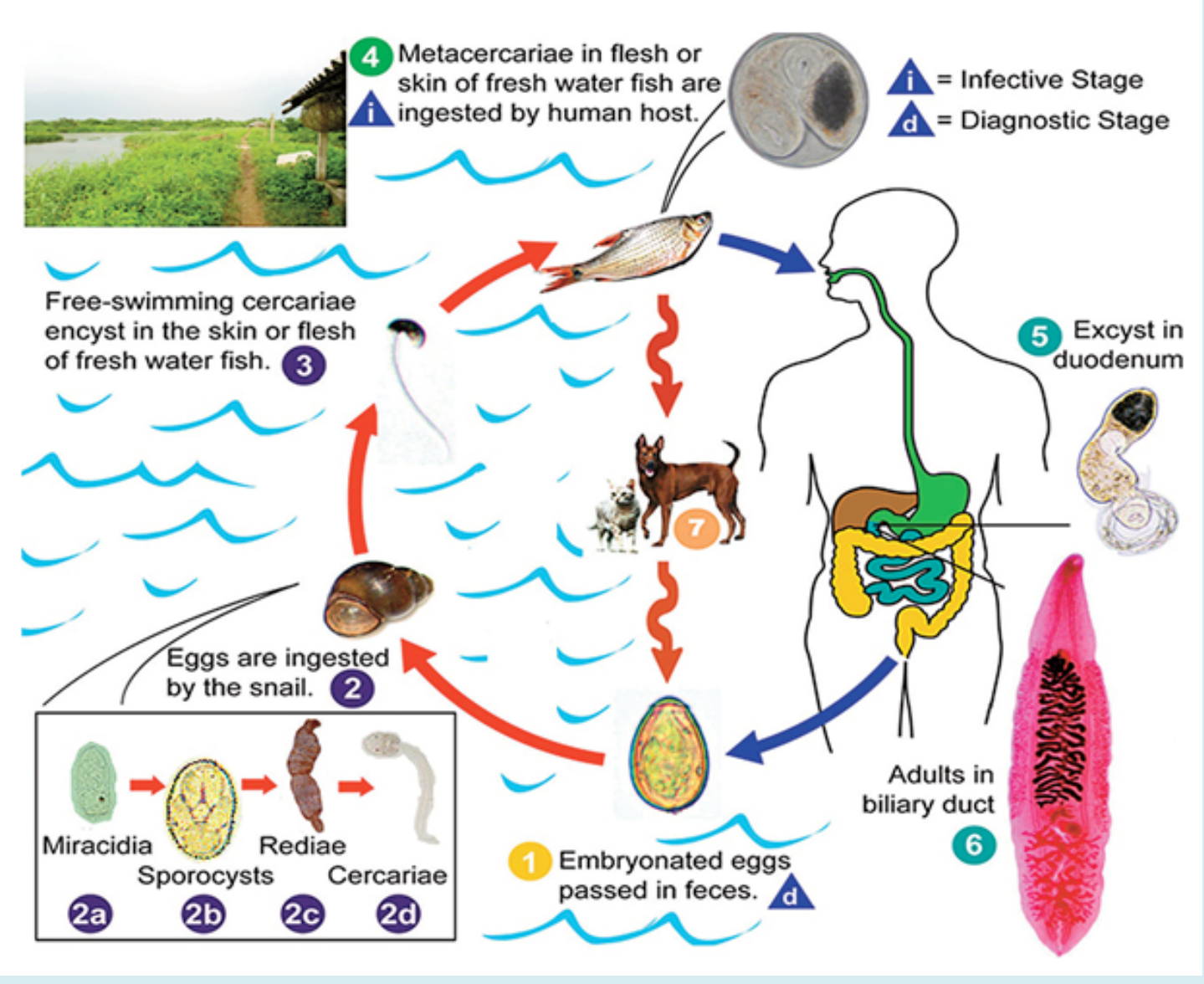

Figure 2: The life cycle of Opisthorchis felineus as a representative for Fish-borne Zoonotic Trematode (FZT) species [16].

\section{Pathophysiology of Opisthorchiasis-Associated Cholangiocarcinoma}

Acute inflammation of the hepatic bile ducts and portal connective tissue are the first effect of acute infection caused by Opisthorchiasis [17]. However, several studies on animal models have proven that multiple stages of carcinogenesis is an offshoot of chronic infections and inflammation. For example, In a model of experimental infection of a hamster with $O$. felineus, there was a clear cut evidence of precancerous lesions, e.g., inflammation, fibrosis, and biliary intraepithelial neoplasia of up to the III grade $[17,18]$.
In long term infections and inflammations, histopathologically changes such as hyperplasia, adenomatous formations, and granulomatous inflammation can be seen in the bile duct epithelium via different microscopic methods [19]. The aetiology for these is due to mechanical damage and metabolic products such as oxysterol, catechol estrogen quinone-like etc excreted by the liver flukes which are injurious to the cell of the host. Mechanical injury occurs during the processes of feeding and migration, leading to ulceration of the periductal tissue. This allows for the infiltration of fluke eggs into the tissue resulting in granulomatous inflammation of the tissue 
around them. Hyperplasia of the bile duct epithelium may be induced as a result of interaction with fluke metabolic products which may be immunogenic or toxic [19-31].

Infiltration of inflammatory cells has been linked to the presence of specific antigens of the liver flukes [29]. It's been hypothesized that this inflammation could be mediated by parasite-specific inflammatory cytokine interleukin (IL)-6 and later it was demonstrated that elevated IL-6 to $0 . s p p$ excretory/secretory substance levels were linked to an increased risk of $63 \%$ for developing advanced periductal fibrosis (APF) and that individuals in the third quartile of IL-6 production had a $127 \%$ higher risk of developing advanced periductal fibrosis (APF) than individuals in the first quartile of IL-6 production [4]. It is now well established that chronic inflammation caused by long-term or recurrent 0 . spp infections plays a large part in the development of many of the factors and events that lead to cholangiocarcinoma, most notably due to the surge of production of nitric oxide (NO) [20,26-38].

Reactive nitrogen species, such as nitric oxide (NO), and reactive oxygen species such as superoxide $\left(\mathrm{O}_{2}^{-}\right)$are produced in chronically inflamed tissues (using inducible NO synthase, or iNOS, in the case of NO) as an immune response aimed at killing the parasite [20,24-26]. Immune mediated response to the parasites activate cells such as neutrophils, eosinophils and macrophages in the early stage while mononuclear subtypes including lymphocytes, macrophages, plasma cells and mast cells to synthesize NO in the later stages, which is both cytotoxic and genotoxic [24-31]. NO can react with $\mathrm{O}_{2}{ }^{-}$ to produce $\left(\mathrm{ONOO}^{-}\right)$peroxynitrate, a more reactive molecule that can cause oxidative and nitrative DNA damage through the formation of DNA adducts such as 8-oxo-7,8-dihydro-2'deoxyguanosine (8oxodG) and 8-nitroguanine [4].

This resultant DNA damage has been linked to an indirect cause of carcinogenesis via an increase in cell proliferation caused by proliferating cell nuclear antigen (PCNA) accumulation in the bile duct epithelium (more common with recurrent infections) [31-38]. As well as a direct causal link between NO and carcinogenesis through inhibiton of DNA repair and apoptosis. Bursts of NO produced, as a result of chronic opisthorchiasis infection and inflammation, may also increase the potential for the endogenous synthesis of N-nitrosamines, such as the carcinogenic N-dimethylnitrosamine (NDMA) [24-34]. This reaction occurs within the inflamed bile duct [24]. NDMA levels is a well known biomarker as it is often detected in the urine of infected individuals.

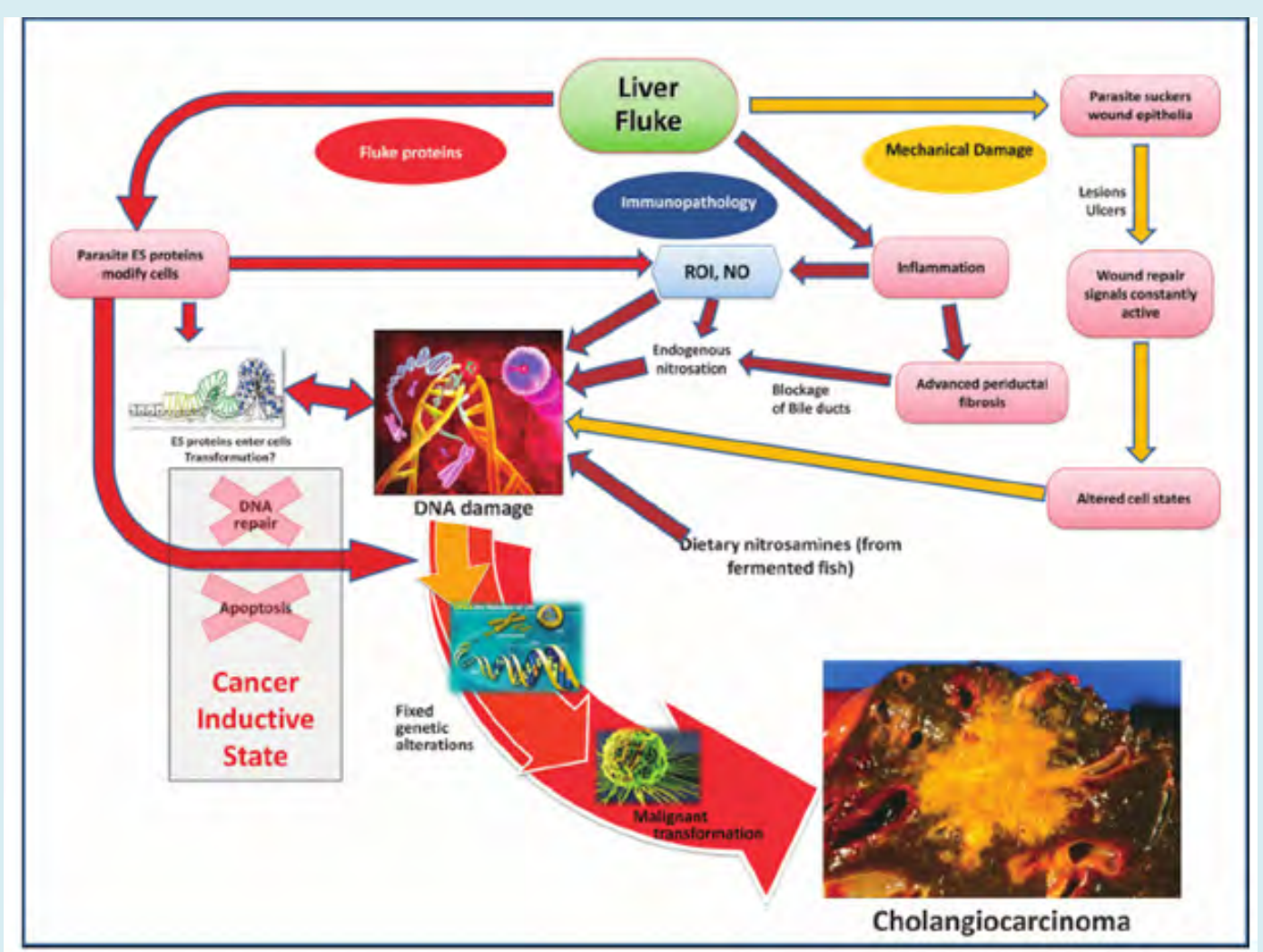

Figure 3: Hypothesized pathways of pathogenesis of opisthorchiasis-associated cholangiocarcinoma. Adapted from ref [36]. 


\section{Public Health Open Access}

Lipid peroxidation (LPO) is another effect of inflammation-linked ROS/RNS [20,35]. The harmful effects of lipid peroxidation can be linked to cytotoxic and genotoxic properties of the secondary aldehyde species (4- Hydroxynonenal (HNE) and Malone dialdehyde (MDA) that result from lipid peroxidation chain reactions. Products such as etheno-DNA adducts ( $\varepsilon \mathrm{dA}) 1, \mathrm{~N}^{6}$-etheno$2^{\prime}$-deoxyadenosine and $(\varepsilon \mathrm{dC}) 3, \mathrm{~N}^{4}$-etheno-2'-deoxycytidine which are products of the reaction of DNA bases with the oxidized lipids (such as trans-4-hydroxy-2-nonenal) [33-38]. These oxidative lesions can initiate carcinogenesis through specific base pair substitutions and have been shown to serve as biomarkers in the leukocytes of Opisthorchiasis infected individuals, alongside 8-oxodG [38] (Figure 3).

\section{Diagnosis}

Detection of fluke eggs in stools represent the gold standard method and definitive diagnosis of opisthorchiasis However, it's becoming increasingly unreliable in cases of low worm burden. More than one stool sample may be needed to identify the eggs. The eggs of Opisthorchis are very similar to those of Clonorchis, another liver fluke, but can be distinguished by microscopic features. This is because the method relies on the parasites having reached maturity level required to release eggs into the gastrointestinal tract, which leads to a low specificity as eggs may not be present despite an infection [4].

Monoclonal antibodies enzyme linked immunosorbent assay have been used to detect $O$. spp antigens in the feces, or coproantigens, and have been proven to be more sensitive than microscopy as it does not cross-react with flukes, when compared with indirect antibody enzyme linked immunosorbent assay, but it only detects current infections, while indirect antibody ELISA can detect past infections, as antibodies persist in body fluids following treatment with praziquantel. Indirect antibody ELISA is used when only a blood or urine sample is available. While antibody levels are higher in serum, urine is a noninvasive alternative that does not require rapid processing or a trained phlebotomist [21].

Molecular detection on faecal samples by Polymerase Chain Reactor have been described in different researches in several parts of the World. At the Tomsk Opisthorchiasis Consortium (TOPIC) held in Tomsk, Russian Federation [2]. Two molecular techniques were highlighted which includes the Taqman-based real-time PCR which was reported to have a high degree of specificity and sensitivity when working with faeces collected from experimental animal models infected with local strains of $O$ felineus. This is similar to results obtained from the molecular diagnosis of $O$. viverrini and $C$. sinensis in different studies and reliable diagnostic differentiation of $O$. felineus infection. Providing point of care diagnostic assays is still a vision for healthcare researchers [2].

Loop-mediated isothermal amplification (LAMP) method is the second molecular diagnostic techniques that have been proven to be faster when compared to the traditionally used PCR, it is sensitive, specific and requiring minimal processing and instrumentation, with results available by reading with the naked eye. This method has been used for the detection of the $O$. viverreni and $C$. Sinensis and its application to detection of $O$. felineus is being established [2].

Additionally, use of imaging techniques such as Ultrasound, Computer Tomography (CT) and Magnetic Resonance Imaging (MRI) can sometimes reveal cysts containing the parasite. Imaging methods are also used to determine the extent of the hepatobiliary disease [20]. Both CT and MRI can determine malignancy, but MRI provides superior soft-tissue resolution, can determine periductal infiltration and the extent of localization of a tumor, and can identify small lesions to a higher degree than CT. An endoscopic retrograde cholangiopancreatography (ERCP) is used to image the biliary tree while also obtaining biliary brushings and bile samples for histopathology and cytology. Magnetic resonance cholangiopancreatography (MRCP) is a magnetic resonance imaging scan optimized for the biliary and pancreatic ducts and is increasingly preferred as the diagnostic test of choice due to its noninvasive nature as opposed to ERCP. All current available biomarkers lack specificity and sensitivity [4].

\section{Treatment}

The main treatment for Opisthorchiidae is based on giving praziquantel at $25-40 \mathrm{mg} / \mathrm{kg}$ body weight [17] some researchers have suggested the use of albendazole at 10 $\mathrm{mg} / \mathrm{kg}$ body weight. Praziquantel is marketed under many brand names depending on the manufacturer, for example Biltricide and Droncid from Bayer for clinical and veterinary uses, respectively. Data from studies on praziquantel in the 0 . felineus animal model and in vitro on juvenile and adult worms, have revealed the effectiveness of the praziquantel drug. The mode of action of this drug is that it disrupts calcium ion homeostasis and antagonizes voltage-gated calcium channels leading to distortion of parasite motility, viability, tegument damage as well as death of the parasite $[1,2,4,5]$.

However, research have shown that without praziquantel treatment, 8-nitroguanine and 8-oxodG were shown to remain present in the epithelium of hamsters six months after they were infected [20]. The amount of the DNA adducts is also significantly lowered by antiparasitic drug treatment and can also be measured in the urine [4]. 


\section{Public Health Open Access}

However, reinfection was shown to increase the rate of inflammatory cell infiltration, iNOS expression in the epithelium, production of NO, and formation of 8-nitroguanine and 8oxodG with each reinfection-ultimately increasing DNA damage and risk of cancer development [4]. This raises the question as to whether or not it is best to treat individuals who are at a high risk of reinfection with praziquantel, as it could do more harm than good. There are many societal, ethical and legal complications to this question. However, until this question is answered, it is suggested that praziquantel must be given to only those who are not at risk of reinfection [4].

\section{Prevention}

The campaign for reduction of raw fish consumption among the aboriginal population of Western Siberia to date has proven ineffective and difficult to implement in practice as its always met with stiff resistance as inherent culture is really difficult to change. This is further complicated by the high sensitivity and specificty detecting infected cases that invariably have light infection ( $\mathrm{egg} \mathrm{g}^{-1}$ feces $<1000$ ) as well as the problem of reinfection highlighted earlier. Despite the huge amount of people infected and at risk of infection, in addition to the economic losses resulting from liver fluke infection, require the devotion of time and resources to develop and implement control and prevention measures. Ideally, improvements in hygiene and sanitation practices prevent transmission of eggs from feces, which disrupts the cycle of fluke maturation and transmission. To be successful and long-lasting, prevention programs require broad community acceptance and participation.

The easiest and most promising way of preventing opisthorchiasis is decontaminating fish, as metacercariae are theonly source of infection for humans. A multitude of studies have examined the viability of metacercariae under various environmental conditions and it is known that they remain viable when frozen at $-20^{\circ} \mathrm{C}$ for up to 30 days. Temperatures of $>50^{\circ} \mathrm{C}$ kill metacercariae in a few minutes, however, It is generally recommended to cook fish in boiled water for more than 30 mins to ensure that metaccercariae are killed in frozen pieces of fish they must be cooked for a long time. Very popular fish dishes of dried and salted fish are not safe for consumption. Salted fish is generally considered ready in 24$48 \mathrm{hrs}$. It has been proven that metacercariae remain viable under high salt concentrations for up to 14 days. Therefore, in this regard, the popular dish of the Western Siberia in Russia and eastern Europe of slightly salted fish is not safe at all. The same is true for dried fish where greater than 12 days of drying are required to kill $99 \%$ of meta-cercariae. Given that the weight of fish and the temperature of drying are not constant even at fish plants or after 12-day period, dried fish remain dangerous for consumption. Hot smoking is a reliable method of fish decontamination. A period of 2-2.5 hrs at 70- $80^{\circ} \mathrm{C}$ kills all metacercariae. Alternatively, cold smoking has similar effects to those of drying and salting. The same is true for treating fish with vinegar where it has been shown that $100 \%$ of metacercariae remain viable and infectious after 24 hrs.

\section{Control}

Interrupting the life cycle of the parasite has always been regarded as a promising way of disease control, for example application of molluscicides to control snail populations. Low concentrations of certain molluscicides (e.g., phenasal and niclosamide) are lethal for infected snails, sublethal for uninfected ones, and presumably nontoxic forother animals. Before the discovery of praziquantel anti-helminthic activity, application of molluscicides was practiced for some time in Russia. The disadvantage of this approach is it's adverse effect on the ecology of the water body. The economic feasibility of using molluscicides is only practical for small water bodies since the costs of treating big areas are extremely high and do require repeated treatments as it has been shown that snail populations are restored in about 5 years, Therefore, decontamination of aquatic bodies has been abandoned in Russia [9].

The control efforts are primarily focused on the reduction and elimination of parasite transmission by ensuring proper food preparation, promoting the development of improved diagnostic techniques, providing chemotherapy, and improving sanitation. A combination of health education, mass treatment, and governmental aid could significantly reduce liverfluke infection. Emphasis on health education is also been placed on the younger generation and foreigners in school as a part of the conventional curriculum. So far, this has been very effective as there are increasing number of foreign students in the Western Siberian region particularly in Tomsk region which play hosts to seven Universities, most of which have different program for International students and has no known record of any of International students suffering from Opisthorchiasis.

The possibility of developing a vaccine against liver fluke is not such a easy task but this approach is been pursed by researchers although no success has been recorded thus far. The genome mapping for all the Liver Flukes is expected to facilitate vaccine development and tests for it's control. To ensure the success of liver fluke control programs, trans disciplinary approach is required.

\section{Recent Public Health Interventions For $\boldsymbol{O}$. Felineus in Western Siberian Region}

As at present, no continous disease control programme has been developed and implemented in the region by both 


\section{Public Health Open Access}

the regional government of the affected regions (Western Siberian) as well as the central government of the Russian Federation this may not be unconnected with the fact that Opisthorchiasis caused by 0 . felineus is a chronic illness and even assymptomatic in a sizeable number of population [4]. However a lot of researchers of local and international repute are beginning to pay attention to this neglected disease. Hence different models are been proposed including the Social Dynamic Model (SDM) discussed earlier in this article. The programme puts into consideration sociocultural processes that animate the transmission of the 0 . felineus parasite and the specificities of the local context where intervention is planned and not only the biomedical components of the disease. This is similar to such integrated approaches illustrated by; for example the history of opisthorchiasis control in Thailand [39] and by the experiences with interventions for zoonotic tapeworm Taenia solium in Northern Lao People's Democratic Republic [40].

Noteworthy is the attention paid by scientists in the mid part of the last decade from Russia, Southeast Asia, Europe, and the United States who came together in a city in Western Siberia (Russia) to form a Tomsk OPIsthorchiasis Consortium (TOPIC). The initiative was designed to strengthen integrated control, to conduct research and to raise awareness on Opisthorciasis afflicting populations not only in the tropical regions of East Asia but also in temperate and semi-arctic areas of Europe and Asia [1]. The TOPIC consortium adopted the "Lawa model," as a template which has been successfully used previously and is based on using the EcoHealth approach at the lake Lawa region of Northeast Thailand [39]. This method is to be modified according to local health care systems and cultural sensitivities in other endemic areas, but not differing in essence of involving trans-disciplinary and stakeholder participation at every level. This initiative was supported as Private and Public Partnerships. However, there is still much to be done in translating research and theoretical approaches into practical realities in the Public health Intervention for this chronic disease.

\section{Conclusion}

Despite the significant global burden of Opisthorchiasis, it is still one of the most neglected disease probably due to the fact that it is responsible for a chronic illness, however it's time to start putting on the spotlight on it, one of which the publication aims to achieve with much empasis on its distribution in the Western Siberian region.

\section{References}

1. Pakharukova MY, Mordvinov VA (2016) The liver fluke Opisthorchis felineus: biology epidemiology and carcinogenic potential. Trans R Soc Trop Med Hyg
110(1): 28-36.

2. Ogorodova LM, Fedorova OS, Sripa B, Mordvinov VA, Katokhin AV, et al. (2015) Opisthorchiasis: An Overlooked Danger. PLoS Negl Trop Dis 9(4).

3. Pozio E, Armignacco 0, Ferri F, Gomez MMA (2013) Opisthorchis felineus, an emerging infection in Italy and its implication for the European Union. Acta Trop 126(1): 54-62.

4. Thomas H, Connor TO, Anchalee T, Nisana N, Watcharin L, et al. (2017) Opisthorchiasis and cholangiocarcinoma in Southeast Asia: an unresolved problem. Int J Gen Med 10: 227-237.

5. Fedorova OS, Kovshirina YV, Kovshirina AE, Fedotova MM, Deev IA, et al. (2017) Opisthorchis felineus infection and cholangiocarcinoma in the Russian Federation: a review of medical statistics. Parasitol Int 66(4): 365-371.

6. Olga Z, Peter O, Ekaterina AG, Marina MF, Yulia VK, et al. (2018) Life by the river: neglected worm infection in Western Siberia and pitfalls of a one-size-fits-all control approach. Critical Public Health 28(5): 534-545.

7. Gomez MMA, Ludovisi A, Amati M, Pozio E (2013) Validation of an excretory/secretory antigen basedELISA for the diagnosis of Opisthorchis felineus infection in humans from low trematode endemic areas. PLoS One $8(5)$.

8. Sripa B, Kaewkes S, Sithithaworn P, Mairiang E, Laha T, et al. (2007) Liver fluke induces cholangiocarcinoma. PLoS Med 4(7).

9. Sithithaworn P. Andrews, R. Khon Kaen University, Khon Kaen, Thailand SV Shekhovtsov, et al. [Eds.], Elsevier, pp: 170-178.

10. Mordvinov VA, Yurlova NI, Ogorodova LM, Katokhin AV (2012) Opisthorchis felineus and Metorchis bilis are the main agents of liver fluke infection of humans in Russia. Parasitol Int 61(1): 25-31.

11. Petney TN, Andrews RH, Saijuntha W, Wenz Mucke A, Sithithaworn P (2013) The zoonotic, fish-borne liver flukes Clonorchis sinensis, Opisthorchis felineus and Opisthorchis viverrini. Int J Parasitol 43(12-13): 10311046.

12. Maksimova GA, Pakharukova MY, Kashina EV, Zhukova NA, Kovner AV, etal. (2017) Effect of Opisthorchis felineus infection and dimethylnitrosamine administration on the induction of cholangiocarcinoma in Syrian hamsters. Parasitol Int 66(4): 458-463. 


\section{Public Health Open Access}

13. (IARC) IARC working group on the evaluation of carcinogenic risks to humans (2012) Biological agents. A review of human carcinogens. In: IARC Monographs on the Evaluation of Carcinogenic Risks to Humans 100: $1-441$.

14. Bouvard V, Baan R, Straif K, Grosse Y, Secretan B, et al. (2009) A review of human carcinogens Part B: biological agents. The Lancet Oncology 10(4): 321-322.

15. de Martel C, Ferlay J, Franceschi S, Vignat J, Bray F, et al. (2012) Global burden of cancers attributable to infections in 2008: a review and synthetic analysis. Lancet Oncol 13(6): 607-615.

16. Jongsuksuntigul $P$, Imsomboon $T$ (2003) Opisthorchiasis control in Thailand. Acta Trop 88(3): 229-232.

17. Zen Y, Adsay NV, Bardadin K, Colombari R, Ferrell L, et al. (2007) Biliary intraepithelial neoplasia: an international interobserver agreement study and proposal for diagnostic criteria. Mod Pathol 20(6): 701-709.

18. Tsokos CG, Krings G, Yilmaz F, Ferrell LD, Gill RM (2016) Proliferative index facilitates distinction between benign biliary lesions and intrahepatic cholangiocarcinoma. Hum Pathol 57: 61-67.

19. Pungpak S, Chalermrut K, Harinasuta T, Viravan C, Schelp PF, et al. (1994) Opisthorchis viverrini infection in Thailand: symptoms and signs of infection-a populationbased study. Trans R Soc Trop Med Hyg 88(5): 561-564.

20. Bhamarapravati N, Thammavit W, Vajrasthira S (1978) Liver changes in hamsters infected with a liver fluke of man, Opisthorchis viverrini. Am J Trop Med Hyg 27(4): 787-794.

21. Mantovani A, Allavena P, Sica A, Balkwill F (2008) Cancerrelated inflammation. Nature 454(7203): 436-444.

22. Holzinger F, Z'Graggen K, Buchler MW (1999) Mechanisms of biliary carcinogenesis: a pathogenetic multi-stage cascade towards cholangiocarcinoma. Ann Oncol 10(4): 122-126.

23. Ohshima H, Bartsch H (1994) Chronic infections and inflammatory processes as cancer risk factors: possible role of nitric oxide in carcinogenesis. Mutat Res 305(2): 253-264.

24. Inoue S, Kawanishi S (1995) Oxidative DNA damage induced by simultaneous generation of nitric oxide and superoxide. FEBS Lett 371(1): 86-88.

25. Kawanishi S, Hiraku Y (2006) Oxidative and nitrative DNA damage as biomarker for carcinogenesis with special reference to inflammation. Antioxid Redox Signal 8(5-6): 1047-1058.

26. Yongvanit P, Pinlaor S, Bartsch H (2012) Oxidative and nitrative DNA damage: key events in opisthorchiasisinduced carcinogenesis. Parasitol Int 61(1): 130-135.

27. Pinlaor S, Sripa B, Sithithaworn P, Yongvanit P (2004) Hepatobiliary changes, antibody response, and alteration of liver enzymes in hamsters re-infected with Opisthorchis viverrini. Exp Parasitol 108(1-2): 32-39.

28. Jaiswal M, LaRusso NF, Burgart LJ, Gores GJ (2000) Inflammatory cytokines induce DNA damage and inhibit DNA repair in cholangiocarcinoma cells by a nitric oxidedependent mechanism. Cancer Res 60(1): 184-190.

29. Jaiswal M, LaRusso NF, Shapiro RA, Billiar TR, Gores GJ (2001) Nitric oxide-mediated inhibition of DNA repair potentiates oxidative DNA damage in cholangiocytes. Gastroenterology 120(1): 190-199.

30. Torok NJ, Higuchi H, Bronk S, Gores GJ (2002) Nitric oxide inhibits apoptosis downstream of cytochrome C release by nitrosylating caspase 9. Cancer Res 62(6): 1648-1653.

31. Srivatanakul P, Ohshima H, Khlat M, Parkin M, Sukaryodhin S, et al. (1991) Opisthorchis viverrini infestation and endogenous nitrosamines as risk factors for cholangiocarcinoma in Thailand. Int J Cancer 48(6): 821-825.

32. Satarug S, Haswell EMR, Tsuda M, Mairiang P, Sithithaworn P, et al. (1996) Thiocyanate-independent nitrosation in humans with carcinogenic parasite infection. Carcinogenesis 17(5): 1075-1081.

33. Satarug S, Haswell EMR, Sithithaworn P, Bartsch H, Ohshima H, et al. (1998) Relationships between the synthesis of $\mathrm{N}$-nitrosodimethylamine and immune responses to chronic infection with the carcinogenic parasite, Opisthorchis viverrini, in men. Carcinogenesis 19(3): 485-491.

34. Dechakhamphu S, Pinlaor S, Sitthithaworn P, Nair J, Bartsch $\mathrm{H}$, et al. (2010) Lipid peroxidation and etheno DNA adducts in white blood cells of liver fluke-infected patients: protection by plasma alpha-tocopherol and praziquantel. Cancer Epidemiol Biomarkers Prev 19(1): 310-318.

35. Pinlaor S, Hiraku Y, Yongvanit P, Oikawa ST, Ma N, et al. (2006) iNOS-dependent DNA damage via NF-kappaB expression in hamsters infected with Opisthorchis viverrini and its suppression by the antihelminthic drug 
praziquantel. Int J Cancer 119(5): 1067-1072.

36. Sripa B, Kaewkes S (2000) Relationship between parasite-specific antibody responses and intensity of Opisthorchis viverrini infection in hamsters. Parasite Immunol 22(3): 139-145.

37. Chotigeat W, Ruenwongsa P (1986) Types of collagen in Opisthorchis viverrini infected hamster liver. Mol Biochem Parasitol 18(3): 377-387.

38. Mairiang E, Elkins DB, Mairiang P, Chaiyakum J, Chamadol N, et al. (1992) Relationship between intensity of Opisthorchis viverrini infection and hepatobiliary disease detected by ultrasonography. J Gastroenterol Hepatol 7(1): 17-21.

39. Sripa B, Tangkawattana S, Laha T, Kaewkes S, Mallory FF, et al. (2015) Toward integrated opisthorchiasis control in Northeast Thailand: The Lawa Project. Acta Trop 141: 361-367.

40. Bardosh K, Inthavong P, Xayaheuang S, Okello AL (2014) Controlling parasites, understanding practices: the biosocial complexity of a One Health intervention for neglected zoonotic helminths in northern Lao PDR. Soc Sci Med 120: 215-223. 